\title{
Determining the role of radiotherapy in the adjuvant management of gastric cancer: an ocean apart
}

\author{
Benjamin W. Corn • Ravit Geva
}

Published online: 20 February 2010

(C) Springer-Verlag 2010

\begin{abstract}
Although the incidence of gastric cancer may be leveling off as of late, mortality rates-especially among those with locally advanced disease-are quite formidable [1]. The cornerstone of therapy for locally advanced disease continues to be surgical extirpation but a consensus has emerged that resection should be supplemented with adjuvant therapies which must continually be improved in order to optimize the percentage of patients who achieve cure. The agents that should comprise such adjuvant programs have been debated for well over a decade.
\end{abstract}

At present, clinicians who counsel patients diagnosed with locally advanced gastric adenocarcinoma are ironically left with two standards of care; neither of which is associated with cure in the majority of cases. The first option is to follow the schema of Intergroup Study 0116 where chemoradiation (fluorouracil and leucovorin plus 45 Gy delivered to the surgical bed and draining lymph nodes) favorably influenced disease-free survival and overall survival [2]. The second alternative is to invoke the results published by Cunningham and colleagues [3] who observed that peri-operative chemotherapy significantly improved progression-free and overall survival among patients with operable disease. This experience, often referred to as the MAGIC study, involved administration of epirubicin, cisplatin and fluorouracil (Three pre-operative cycles; three post-operative cycles of ECF) and begged the question of radiotherapy by excluding the latter modality from trial design.

\footnotetext{
B. W. Corn $(\bowtie) \cdot$ R. Geva

Institute of Radiotherapy and Department of Oncology,

Tel Aviv Medical Center of Tel Aviv University School

of Medicine, 6 Weizman Street, Tel Aviv, Israel

e-mail: bencorn@tasmc.health.gov.il
}

In this issue of the journal, Bamias and colleagues report the results of a phase III trial carried out in Greece that sought to determine the value of radiotherapy in adjuvant regimens for this disease [4]. While Bamias and co-authors must be commended for asking a timely question vis-à-vis the importance of radiotherapy for this disease, their study is wanting from a qualitative perspective [5] in several key aspects.

First, the backbone chemotherapy protocol chosen for this study is puzzling. Although docetaxel has been associated with an increase in the overall survival of metastatic gastric cancer [6], the added value of 2 weeks was greatly questioned by many clinicians especially in light of the side effects (e.g., complicated neutropenia) of this agent. The drug, therefore, has not been adopted as a standard of care by most centers around the world. Moreover, docetaxel has not been compared to the conventional protocols of ECF or $\mathrm{CF}$ in the adjuvant scenario. In addition, the authors themselves acknowledge that exchanging the cisplatin for carboplatin represents a weakness since the two drugs have never been proven to be equivalent, even in the metastatic setting.

Second, the use of adjuvant chemotherapy instead of peri-operative chemotherapy as employed in the MAGIC trial is in itself surprising. Notwithstanding the promising outcome seen with S1 in the Far East [7], phase III trials [8-11] that have attempted to prove the added value of adjuvant chemotherapy to operation alone have generally failed despite clues of an advantage in previous results from meta-analyses [12, 13]. In the MAGIC trial, the valuable component of the protocol was almost certainly the pre-operative chemotherapy period as only $40 \%$ of patients actually proceeded to the post-operative segment.

A third weakness incorporated into the design of Bamias et al. is the decision to add the radiotherapy after the $3 \mathrm{rd}$ 
course of chemotherapy. Such a time interval is well beyond the purported biologic window of opportunity that can be inferred from the Intergroup trial.

Regarding the radiotherapy itself, the investigators failed to mandate the real-time review of radiation portals that is requisite to assure the implementation of reliably administered treatment, even for the unsophisticated AP/ PA fields that were employed by the participating physicians. The importance of the latter was underscored in Intergroup trial 116 (the Gastric Surgical Adjuvant Trial) where $35 \%$ of the radiation fields designed were nullified during rapid review but corrected before the patients received their first cGy [2]. In so doing, Macdonald and colleagues demonstrated benefits in local control and survival among those randomized to the adjuvant arm which included radiotherapy. The notion of real-time review of complex beam apertures with rapid turnover on the part of experts has now been successfully adopted for most tumors of the gastro-intestinal and hepatobiliary systems [14, 15]. Such efforts are facilitated by electronic transmission of image-centric datasets and a commitment on the part of physicians to apply the best available peer-reviewed treatment.

Although the addition of radiotherapy was associated with a (statistically insignificant) halving of local failures in the Greek trial, RT had no impact on survival. Unfortunately, the trial failed to reach the target accrual, and the authors candidly admitted that their results lacked the power to offer any meaningful solution to the question at hand. In a recently reported trial of locally advanced adenocarcinoma of the gastroesophageal junction from Germany [16], the addition of radiotherapy was also associated with a (statistically significant) reduction in local failure among patients but, once again, without translation into a survival benefit. The German trial also did not reach its accrual goals and therefore was not sufficiently powered to evaluate its primary endpoint.

The Cancer and Leukemia Group B (CALGB) recently completed enrollment of over 500 patients to trial 80101 . That study, which directly compares RT plus ECF to RT plus 5-FU/LV among individuals with resected adenocarcinoma of the stomach, endeavors to identify which of these two chemotherapy regimens best prolongs survival while begging the question of radiotherapy (this time by presuming all patients benefit from the use of RT).

Somewhat analogous to the state of affairs with adenocarcinoma of the pancreas, the Atlantic Ocean seems to divide American oncologists (in favor of including irradiation) from European oncologists (in favor of omitting irradiation) when it comes to the adjuvant management of locally advanced adenocarcinoma of the stomach. To bridge this divide in adenocarcinoma of the pancreas, experts from both continents agreed to mount RTOG study
0848 to conclusively assess the contribution of radiotherapy in the adjuvant setting as one of the trial's primary goals. With the advent of new cytotoxic chemotherapies that are not only safe but also active against gastric cancer and the availability of efficient, modern radiotherapeutic plans [17] the time has come to decide, once and for all, whether radiotherapy should be viewed as a mandatory component in the evolving therapeutic strategies for gastric cancer. Although the ChemoRadiotherapy after Induction chemotherapy in Cancer of the Stomach (CRITICS) trial in the Netherlands has been framed with this objective in mind, it is unlikely that the Dutch results will be universally accepted unless there is widespread international participation in a pattern similar to what is expected for protocol 0848. It is hoped that leaders of the respective cooperative oncology groups will harness the equipoise that presently exists lest we remain with capricious whims instead of conclusive recommendations vis-à-vis adjuvant management of resectable gastric cancer at the end of the day.

The global incidence of gastric adenocarcinoma exceeds 500,000 per year. Irrespective of geography, oncologists "can no longer stomach" the absence of Level I evidence to guide them in the optimal adjuvant management of locally advanced gastric cancer.

Conflict of interest statement The authors indicated no potential conflicts of interest.

\section{References}

1. Parkin DM, Bray F, Ferlay J, Pisani P (2005) Global cancer statistics. CA Cancer J Clin 55:74-108

2. MacDonald JS, Smalley SR, Bendetti J et al (2001) Chemoradiotherapy after surgery compared to surgery alone for adenocarcinoma of $t$ he stomach or GE junction. N Engl J Med 10:725730

3. Cunningham D, Allum WH, Stenning S et al (2006) Perioperative chemotherapy versus surgery alone for resectable gastroesophageal cancer. N Engl J Med 355:11-20

4. Bamias A, Karina M, Papakostas P et al (2010) A randomized phase III study of adjuvant platinum/docetaxel chemotherapy with or without radiation therapy in patients with gastric cancer. Cancer Chemother Pharmacol. doi:10.1007/s00280-010-1256-6

5. Bentzen SM (2003) A user's guide to evidence-based oncology. Eur J Cancer Suppl 1(6):77-91

6. Van Cutsem E, Moiseyenko VM, Tjulandin S et al (2006) Phase III study of docetaxel and cisplatin plus fluorouracil compared with cisplatin and fluorouracil as first-line therapy for advanced gastric cancer. A report of the V325 study group. J Clin Oncol 24:4991-4997

7. Sakuramoto S, Sasako M, Yamaguchi T et al (2007) Adjuvant chemotherapy for gastric cancer with $\mathrm{S}-1$, an oral fluoropyrimidine. N Engl J Med 357:1810-1818

8. DeVita F, Giuliani F, Orditura M et al (2007) Adjuvant chemotherapy with epirubicin, leucovorin, 5-fluorouracil, etoposide regimen in resected gastric cancer patients. A randomized phase 
III trial by the Gruppo Oncologico Italia Meridionale (GOIM 9602 Study). Ann Oncol 18:1354-1358

9. Di Costanzo F, Gasperoni S, Manzione L et al (2008) Adjuvant chemotherapy in completely resected gastric cancer. A randomized phase III trial conducted by GOIRC. J Natl Cancer Inst 100:388-398

10. Bouche O, Ychou M, Burtin P et al (2005) Adjuvant chemotherapy with 5-FU and cisplatin compared with surgery alone for gastric cancer. 7 year results of the FFCD randomize phase III trial. Ann Oncol 16:1488-1497

11. Nitti D, Wils J, Guimaraes DS et al (2006) Randomized phase III trials of adjuvant FAMTX or FEMTX compared with surgery alone in resected gastric cancer. A combined analysis of the EROTC GI Group and ICCG. Ann Oncol 17:262-269

12. Mari E, Floriani I, Tinazzi A et al (2000) Efficacy of adjuvant chemotherapy after curative resection for gastric cancer. A metaanalysis of published randomized trials. A study of the GISCAD. Ann Oncol 11:837-843
13. Earle CC, Maroun JA (1999) Adjuvant chemotherapy after curative resection for gastric cancer in non-Asian patients. Revisiting a meta-analysis of randomized trials. Eur J Cancer 35:1059-1070

14. Regine WF, Winter KA, Abrams RA et al (2008) Fluorouracil vs gemcitabine chemotherapy before and after fluorouracil based chemoradiation following resection of pancreatic adenocarcinoma. A randomized controlled trial. JAMA 299:1019-1026

15. Konski A, Kachnic LA (2009) Jury still out on whether advanced technology can improve the outcomes of patients with anal canal cancer. Oncology 23:1092-1096

16. Stahl M, Walz MK, Stuschke M et al (2009) Phase III comparison of pre-operative chemotherapy compared with chemoradiotherapy in patients with locally advanced adenocarcinoma of the esophagogastric junction. J Clin Oncol 27:851-865

17. Soyfer V, Corn BW, Melamud A et al (2007) Three dimensional non-coplanar conformal radiotherapy yields better results than traditional beam arrangements for adjuvant treatment of gastric cancer. Int J Radiat Oncol Biol Phys 69:364-369 